\title{
CRITERIA FOR METRISABILITY
}

\author{
P. J. COLLINS AND A. W. ROSCOE
}

\begin{abstract}
A simple condition on the local bases of a first countable space is shown to imply metrisability, and some new and some well-known metrisation theorems are deduced. Weakening the condition gives new classes of spaces distinct from the class of metrisable spaces.
\end{abstract}

1. Introduction. It is the primary purpose of this paper to present a simple metrisation theorem, give its elementary proof and deduce a number of other metrisation theorems from it. The theorem is

THEOREM 1. In order that a $T_{1}$-space $X$ be metrisable it is necessary and sufficient that for each $x$ in $X$, there be a countable decreasing local neighbourhood basis ${ }^{1}$ $\{W(n, x): n=1,2,3, \ldots\}$ at $x$ satisfying

(A) if $x \in U$ and $U$ is an open set, then there exist a positive integer $s=s(x, U)$ and an open set $V=V(x, U)$ containing $x$ such that $x \in W(s, y) \subseteq U$ whenever $y \in V$.

We conclude by considering conditions weaker than (A) and give examples to show that 'decreasing' cannot be omitted in the statement of Theorem 1 . This gives a class of first countable spaces, distinct from the metrisable spaces, in which every separable space is second countable.

Throughout, $x, y, z$ will denote elements of a space $X$, while $i, j, m, n, r, s$ will be elements of the set $N$ of positive integers. For $A \subseteq X$ and a family $G$ of subsets of $X$, $\operatorname{St}(A, G)$ will denote the union of those elements of $G$ which meet $A$, and $\operatorname{St}(x, G)$ will denote $\operatorname{St}(\{x\}, G) . X$ is developable if it has a development, that is, a sequence $\{G(n): n \in N\}$ of open coverings of $X$ such that, for each $x,\{\operatorname{St}(x, G(n)): n \in N\}$ is a local basis at $x$. For $A \subseteq X, A^{\circ}$ will denote the interior of $A$.

All spaces will satisfy the $T_{1}$ separation axiom unless we specifically allow otherwise.

2. Proof of Theorem 1. The proof of the following lemma is straightforward and is omitted. We shall not require $(C)$ again until the next section, but place it here for convenience.

Received by the editors November 22, 1982 and, in revised form, July 27, 1983.

1980 Mathematics Subject Classification. Primary 54E35; Secondary 54D15, 54D20, 54E15, 54E25, 54E30, 54E65.

'A countable decreasing local neighbourhood basis at $x$ is a sequence $\{W(n, x): n=1,2,3, \ldots\}$ of not necessarily open neighbourhoods $W(n, x)$ of $x$ such that $W(n, x) \supseteq W(n+1, x)$ for each $n$, where the inclusion need not be strict, and such that for each neighbourhood $N$ of $x$ there exists $n$ with $W(n, x) \subseteq N$ 
Lemma 1. Suppose that for each $x$ in a space $X$ there is a decreasing local neighbourhood basis $\{W(n, x): n \in N\}$. Then each of the conditions (B), (C) below is equivalent to (A) of Theorem 1:

(B) for each $x$ and $n$, there exist $r=r(n, x)$, and $s=s(n, x)$ with $r>s>n$, such that $y \in W(r, x)$ implies $x \in W(s, y) \subseteq W(n, x)$;

(C) for each $x$ and $n$, there exists $r=r(n, x)$ with $r \geqslant n$ such that $y \in W(r, x)$ implies $x \in W(n, y)$ and $W(r, y) \subseteq W(n, x)$.

For the rest of the proof of Theorem 1, we shall assume that for each $n$ and $x$, $r(n, x)$ and $s(n, x)$ have been fixed to satisfy (B) and, without loss of generality, such that $r(n, x) \leqslant r(n+1, x)$. Further, we define $r_{1}(n, x)=n$, and inductively,

$$
r_{i+1}(n, x)=r\left(r\left(r_{i}(n, x), x\right), x\right) \text {. }
$$

The next lemma now follows readily from (B).

LEMMA 2. (i) If $x$ belongs to the set $W(r(r(n, x), y), y)$, then that set is contained in $W(n, x)$.

(ii) If $W\left(r_{2}(n, x), x\right)$ meets $W\left(r_{2}(m, y), y\right)$, then either $W\left(r_{2}(n, x), x\right) \subseteq W(m, y)$ or $W\left(r_{2}(m, y), y\right) \subseteq W(n, x)$.

For each $n$, define $G(n)$ to be the open covering consisting, as $x$ varies, of all $W(r(n, x), x)^{\circ}$. Then $\operatorname{St}(x, G(r(n, x))) \subseteq W(n, x)$ by Lemma 2(i). So $\{G(n): n \in$ $N\}$ is a development for $X$.

Putting $V(n, x)=W\left(r_{2 n}(1, x), x\right)^{\circ}$, define a real-valued function $c$ by

$$
c(x, y)= \begin{cases}0 & \text { if } x=y, \\ 1 & \text { if }\{x, y\} \nsubseteq V(1, z) \text { for all } z, \\ 2^{-n} & \text { if there exists } z \text { such that }\{x, y\} \subseteq V(n, z) \\ & \text { and } n \text { is maximal with respect to this relation. }\end{cases}
$$

Putting $H(n)=\{V(n, x): x \in X\}$, we see that $H(n)$ refines $G(n)$ for each $n$, and so $\{H(n): n \in N\}$ is a development for $X$. Hence, $c$ is defined on all of $X \times X$. Further, by Lemma 2(ii), if

$$
V\left(n+1, x_{i}\right) \cap V\left(n+1, x_{i+1}\right) \neq \varnothing \quad \text { for } i=1,2,
$$

then there exists $j \in\{1,2,3\}$ such that

$$
\bigcup_{i=1}^{3} V\left(n+1, x_{i}\right) \subseteq V\left(n, x_{j}\right) .
$$

So, as in the proof of the uniform space metrisation theorem (see, for example, $\mathrm{N}$. Bourbaki [4, pp. 143-144]),

$$
d(x, y)=\inf \left\{\sum_{r=0}^{k-1} c\left(x_{r}, x_{r+1}\right): k \in N, x_{r} \in X, x_{0}=x, x_{k}=y\right\}
$$

is such that $d(x, y) \geqslant c(x, y) / 2$ and so defines a metric which generates the topology on $X$. Since the necessity of the conditions is clear, we have completed the proof of Theorem 1 . 
We conclude this section by giving a short proof of Theorem 1 which depends on the result of R. H. Bing [3, Theorem 10] that a collectionwise normal, developable space is metrisable. All that remains is to show collectionwise normality, the proof of which carries over unchanged to more general situations (see Lemma 3 of §5).

Suppose that $\left\{A_{i}: i \in I\right\}$ is a discrete family of closed sets so that, for each $x$ in $A_{i}$, there is an open neighbourhood $U(x)$ of $x$ such that $U(x) \cap A_{j}=\varnothing$ whenever $j \neq i$. Property (A) finds an open neighbourhood $V(x)$ of $x$ and an integer $s(x, U)$ such that $x \in W(s(x, U), y) \subseteq U$ whenever $y \in V(x)$. But then, since the local bases are decreasing, the family $\left\{U_{i}: i \in I\right\}$, where $U_{i}=\cup\left\{V(x): x \in A_{i}\right\}$, is pairwise disjoint and so, $X$ is collectionwise normal.

We hasten to point out that Bing's theorem depends on the construction of a metric as well as some detailed work on covering theorems.

3. Some corollaries to Theorem 1. The results in this section are in some sense equivalent to Theorem 1 .

THEOREM 2. In order that a space $X$ be metrisable it is necessary and sufficient that, for each $x$ in $X$, there be a countable local neighbourhood basis $\{R(n, x): n \in N\}$ at $x$ satisfying

(D) for each $x$ and $n$, there exists an open $V_{1}$ containing $x$ such that $x \in R(n, y)$ whenever $y \in V_{1}$;

(E) for each $x$ and open $U$ containing $x$, there exist $r$ and open $V_{2}$ containing $x$ such that $R(r, y) \subseteq U$ whenever $y \in V_{2}$.

Proof. The result is established when we have shown the existence of local neighbourhood bases satisfying (C) of Lemma 1. This follows quickly once one defines $W(n, x)=\bigcap\{R(i, x): i=1,2,3, \ldots, n\}$.

If $T$ is a topology on $X$, then $g: N \times X \rightarrow T$ is a $\gamma$-function for $X$ of $\mathrm{R}$. E. Hodel [11] (see W. F. Lindgren and P. J. Nyikos [13, pp. 466-467]) provided

(a) for each $x,\{g(n, x): n=1,2,3, \ldots\}$ is a local basis at $x$;

(b) for each $x$ and $n$, there exists $m=m(n, x)$ such that $y \in g(m, x)$ implies $g(m, y) \subseteq g(n, x)$.

R. Fox [7] has given an example of a space which has a $\gamma$-function but is not quasi-metrisable. We have the following result which follows directly from Theorem 2.

THEOREM 3. In order that $X$ be metrisable it is necessary and sufficient that $X$ have a $\gamma$-function $g$ such that, for each $x$ and $n$, there exists $m=m(n, x)$ such that $x \in g(n, y)$ whenever $y \in g(m, x)$.

W. F. Lindgren and P. Fletcher [12] say that $(X, \mathcal{Q})$ is a local quasi-uniform space if $\mathcal{U}$ is a filter on $X \times X$ satisfying

(a) for each $x \in X, \cap\{U(x): U \in \mathcal{Q}\}=\{x\}$;

(b) for each $x \in X$ and $U \in \mathcal{U}$, there exists $V \in \mathcal{U}$ such that $(V \circ V)(x) \subseteq U(x)$.

The topology of $(X, \mathscr{Q})$ is the collection of all sets $A \subseteq X$ such that, for each $x \in A$, there is some $U \in \mathcal{Q}$ with $U(x) \subseteq A$. Lindgren and Fletcher show that spaces with $\gamma$-functions are precisely those with topologies of local quasi-uniform 
spaces with countable bases. We now state the analogue of Theorem 3 for local quasi-uniform spaces.

THEOREM 4. A local quasi-uniform space ( $X, \mathcal{Q})$ has a metrisable topology provided $\mathcal{Q}$ has a countable basis and, given $x \in X$ and $U \in \mathcal{Q}$, there exists $V \in \mathcal{U}$ with $V(x) \subseteq U^{-1}(x)$.

A nonnegative real-valued function on $X \times X$ is a local metric for $X$ if it is zero only on the diagonal, is locally symmetric, and satisfies the local triangle law of V. W. Niemytzki [22]; that is, if

(1) $d(x, y)=0$ if and only if $x=y$,

(2) given $x$ and $\varepsilon>0$, there exists $\delta>0$ such that $d(y, x)<\varepsilon$ whenever $d(x, y)<$ $\delta$,

(3) given $x$ and $\varepsilon>0$, there exists $\delta>0$ such that $d(x, y)<\varepsilon$ whenever $d(x, z)<\delta$ and $d(z, y)<\delta$.

Each local metric $d$ generates a topology on $X$ : a set $U$ is open if and only if, for each $x \in U$, there exists some $\varepsilon>0$ such that $S(x, \varepsilon) \subseteq U$, where $S(x, \varepsilon)=\{y \in X$ : $d(x, y)<\varepsilon\}$. It is possible to show that in the topology generated by a local metric $d$, the set $S(x, \varepsilon)$ is a neighbourhood of $x$ for each $x \in X$ and $\varepsilon>0$. (It is possible to construct an open set $U$ such that $S(x, \delta) \subseteq U \subseteq S(x, \varepsilon)$, where $\delta$ is chosen by (3) above relative to $x$ and $\varepsilon$.) Given this fact, the following is an easy consequence of Theorem 2.

THEOREM 5. In order that a space $X$ be metrisable, it is necessary and sufficient that there exist a local metric for $X$ which generates the topology on $X$.

The conditions of Theorem 2 are recoverable from Theorem 5. Given countable local neighbourhood bases $\{R(n, x): n \in N\}$, all one needs to do is to ensure, as in the proof of Theorem 2, that the local bases are decreasing and to define a local metric by $d(x, y)=0$ if $x=y$, and otherwise $d(x, y)=1 / n$, where $n$ is the smallest integer such that $y \notin R(n, x)$.

4. Theorem 1 and its corollaries in the context of some well-known metrisation theorems. J. Nagata proved the following theorem using the Alexandroff-Urysohn metrisation theorem [1], the theorem of E. Michael [15] that a space $X$ is paracompact if every open covering of $X$ has a $\sigma$-cushioned open refinement, and the theorem of A. H. Stone [26] that paracompactness implies full normality in the category of Hausdorff spaces. We now derive the same result directly from Theorem 1 .

TheOREM 6 (J. NAGATa [21], SEE Also [20]). In order that a space $X$ be metrisable, it is necessary and sufficient that, for each point $x$ of $X$, there exist sequences $\left\{U_{n}(x)\right.$ : $n \in N\}$ and $\left\{V_{n}(x): n \in N\right\}$ of neighbourhoods of $x$ such that

(i) $\left\{U_{n}(x): n \in N\right\}$ is a local neighbourhood basis at $x$,

(ii) $y \notin U_{n}(x)$ implies $V_{n}(y) \cap V_{n}(x)=\varnothing$,

(iii) $y \in V_{n}(x)$ implies $V_{n}(y) \subseteq U_{n}(x)$.

Proof. Necessity being straightforward, we prove sufficiency only. With no loss of generality, we assume that, for each $x$, the neighbourhoods $V_{n}(x)$ decrease with $n$. 
Given $x$ in open $U$, find $n$ such that $U_{n}(x) \subseteq U$. Put

$$
W(n, x)=\bigcup\left\{V_{n}(y): x \in V_{n}(y)\right\} \text {. }
$$

If $m^{\prime}$ is the least positive integer such that $U_{m^{\prime}}(x) \subseteq V_{n}(x)$, set $m=\max \left\{n, m^{\prime}\right\}$. It is straightforward to verify that $x \in W(m, y) \subseteq U_{n}(x)$ whenever $y \in\left(V_{m}(x)\right)^{\circ}$. Thus the conditions of Theorem 1 have been satisfied.

It should not surprise the reader that it is possible to derive Theorem 1 from Theorem 6. This may be done, using the notation of (B), by setting $V_{n}(x)=$ $W(r(r(n, x), x), x)$ and

$$
U_{n}(x)=W(n, x) \cup \bigcup\{W(r(n, y), y): x \in W(r(n, y), y)\} .
$$

The reader will probably find this derivation somewhat harder and longer than the derivation of Theorem 6 above.

In [21], J. Nagata deduced a number of well-known theorems from Theorem 6 . We now give two examples, each of which can be derived very quickly from Theorem 1 or from Theorem 2.

Theorem 7 (Nagata-Smirnov Metrisation Theorem $[19,25])$. A necessary and sufficient condition for a regular space $X$ to be metrisable is that $X$ have a $\sigma$-locally finite basis.

Proof. Necessity can be proved as usual by using the paracompactness of metric spaces. (There are a number of simple direct proofs of this result; see for example M. E. Rudin [24].)

Suppose that $\cup\{G(n): n \in N\}$ is a basis for the topology on $X$, where each $G(n)$ is a locally finite family. There is no loss of generality in assuming that $G(n) \subseteq$ $G(n+1)$ for each $n$. Define $W(n, x)=\bigcap\{\bar{G}: x \in G \in G(n)\}$ and $V(n, x)=$ $\cap\{G: x \in G \in G(n)\}-\cup\{\bar{G}: G \in G(n), x \notin \bar{G}\}$. Since $X$ is regular and $G(n)$ is locally finite, $V(n, x)$ is open and $\{W(n, x): n=1,2, \ldots\}$ is a (decreasing) local neighbourhood basis at $x$. One can then verify that $x \in W(n, y) \subseteq W(n, x)$ whenever $y \in V(n, x)$. So, by Theorem $1, X$ is metrisable.

TheOREM 8 (A. H. Frink [8]). A necessary and sufficient condition for a space $X$ to be metrisable is that, for each $x$ in $X$, there be a local basis $\{P(n, x): n \in N\}$ at $x$ such that, if $x \in X$ and $n \in N$, then there exists a positive integer $m=m(n, x)$ such that $P(m, y) \subseteq P(n, x)$ whenever $P(m, y) \cap P(m, x) \neq \varnothing$.

Rather than prove Theorem 8, we now derive a more powerful result from which it may be immediately deduced as a corollary.

Theorem 9 (ARhangel'Skil-Stone Metrisation Theorem $[2,27]$, SeE also R. L. MOORE [17]). In order that $X$ be metrisable, it is necessary and sufficient that there exist a sequence of open coverings $\{G(n): n \in N\}$ such that, if $U$ is a neighbourhood of a point $x$, there is a neighbourhood $V$ of $x$ and an integer $n$ with $\operatorname{St}(V, G(n)) \subseteq U$.

Proof. Necessity is clear, and sufficiency follows directly from Theorem 2 once one sets $R(n, x)=\operatorname{St}(x, G(n))$. 
The Alexandroff-Urysohn Metrisation Theorem [1] may be deduced in exactly the same way.

J. Williams derives the Nagata-Smirnov Theorem from a metrisation theorem for locally uniform spaces which he proves using the uniform space metrisation theorem. His theorem is now a direct consequence of Theorem 4.

Theorem 10 (J. Williams [29] AND compare Results of V. W. NiemytzKi [22], K. Morita [18] AND J. SUZUKi [28]). A separated local uniformity with a countable basis has a metrisable topology.

Among other results which can be easily deduced from the theorems of $\S \S 1$ and 3 are S. Hanai's theorem [9] that the perfect image of a metrisable space is metrisable, and the fact that P. Roy's space [23] demonstrating the inequivalence of certain dimension functions is metrisable (see [5]).

5. Weakening condition (A): results and counterexamples. In $\S \S 1-3$ we gave several conditions on local bases for a space $X$ which ensure metrisability. In this section we investigate some related but weaker conditions which shed some further light on our main results.

First we give two examples to illustrate the necessity of our assumption in Theorem 1 that the local neighbourhood bases be decreasing.

EXAMPLE 1. Let $X=N \cup\{x, y\}$, where $N$ is the set of positive integers and $x, y$ are two distinct points neither of which is an integer. Give $X$ the topology consisting of all subsets of $N$ together with all cofinite subsets of $X$. This topology can be given local bases as follows:

$$
\begin{aligned}
W(n, x) & =\{x, n, n+1, n+2, \ldots\}, \\
W(n, y) & =\{y, n, n+1, n+2, \ldots\}, \\
W(2 n, r) & =\{x, 2 n, 2 n+1,2 n+2, \ldots\} \quad \text { if } 2 n \leqslant r ; \\
W(2 n, r) & =\{r\} \quad \text { if } 2 n>r, \\
W(2 n-1, r) & =\{y, 2 n-1,2 n, 2 n+1, \ldots\} \quad \text { if } 2 n-1 \leqslant r ; \\
W(2 n-1, r) & =\{r\} \quad \text { if } 2 n-1>r .
\end{aligned}
$$

It is easily verified that these bases satisfy condition (A) without being decreasing, and that $X$ is $T_{1}$ but not $T_{2}$ and is thus not metrisable. In fact, it is easy to see that every second countable space has (possibly nondecreasing) bases satisfying (A).

EXAMPLE 2. Let $X$ be the Michael line [16] formed by scattering the irrationals in the usual topology on the set of real numbers. This space is not metrisable. It can, however, be given nondecreasing local bases satisfying condition (A):

$$
\begin{array}{ll}
W(n, x)=\left(x-\frac{1}{n}, x+\frac{1}{n}\right) & \text { if } x \in Q \text { (the set of rational numbers), } \\
W(1, x)=\{x\} & \text { if } x \notin Q, \\
W(n, x)=\left(x-\frac{1}{n}, x+\frac{1}{n}\right) & \text { if } x \notin Q \text { and } n>1 .
\end{array}
$$


It is interesting to note that the first example is developable but not collectionwise normal, and that the reverse is true of the second. Note also that in each case the bases are eventually decreasing, and in the second case the iocal bases $W(x)$ are chains, in that if $X, Y \in W(x)$, then either $X \subseteq Y$ or $Y \subseteq X$.

Note that the Sierpinski two-point space is a $T_{0}$-space with local bases satisfying (A) which is not $T_{1}$, and that a $T_{0}$-space with countable local neighbourhood bases satisfying (D) and (E) of Theorem 2 is necessarily $T_{1}$.

The next result gives two properties implied of spaces which have (possibly nondecreasing) local neighbourhood bases satisfying a generalisation of property (A).

Lemma 3. Suppose that $X$ is a space and that, for each $x \in X, W(x)$ is a family of subsets of $X$, each containing $x$, satisfying

(F) if $x \in U$ and $U$ is an open set, then there exists an open set $V$ containing $x$ such that whenever $y \in V$ there is some $W \in W(y)$ satisfying $x \in W \subseteq U .^{2}$ Then

(i) if $W(x)$ is a chain for each $x$, then $X$ is collectionwise normal,

(ii) if $W(x)$ is, for each $x$, a countable set of open sets, then $X$ is second countable if it is separable.

Proof. The proof of (i) is exactly as in the alternative proof of Theorem 1 . (Indeed it can readily be verified that under the conditions of (i), a space must necessarily be monotonically normal ${ }^{3}$ (see R. W. Heath, D. J. Lutzer and P. L. Zenor [10]).) When each $W(x)$ is a countable local basis of open sets and $X$ is separable it is easy to show that $\cup\{W(x): x \in D\}$ is a countable basis, where $D$ is a countable dense subset of $X$.

Consider the following condition on a space $X$ which has countable decreasing local neighbourhood bases $\{W(n, x): n \in N\}$ :

(G) if $x \in U$ and $U$ is an open set, then there exists an open set $V$ containing $x$ such that whenever $y \in V$ there is some positive integer $s=s(x, y, U)$ satisfying $x \in W(s, y) \subseteq U$.

This condition implies paracompactness, but the "bow-tie" space of L. F. McAuley [14] shows that it does not ensure metrisability. ${ }^{4}$

We will now turn our attention to the conditions of Theorem 2. For a space to satisfy the conditions of Theorem 2 it must have local neighbourhood bases which satisfy the two conditions (D) and (E) simultaneously. Observe that a space has countable local bases satisfying (E) if and only if it has a $\gamma$-function. We will shortly find a similar characterisation of spaces with bases satisfying (D). That the two conditions need to be satisfied simultaneously is illustrated by Example 1. The bases

\footnotetext{
${ }^{2}$ Every space, of course, has local neighbourhood bases $W(x)$ satisfying $(\mathrm{F})$.

${ }^{3}$ Essentially we owe this remark to A. J. Ostaszewski, whom the first author would like to thank for some useful conversations.

${ }^{4}$ The authors, G. M. Reed and M. E. Rudin, have proved that the existence of local bases satisfying (G), with each $W(n, x)$ open, necessitates $X$ being metric.
} 
which we originally gave may be seen to satisfy (E), and the following bases satisfy (D):

$$
\begin{gathered}
V(n, x)=W(n, x), \quad V(n, y)=W(n, y) \\
V(n, r)=\{x, y, n, n+1, n+2, \ldots\} \quad \text { if } n \leqslant r, \quad V(n, r)=\{r\} \quad \text { if } n>r .
\end{gathered}
$$

We have already seen that this space is not metrisable.

It is noteworthy that, if $\{G(n): n \in N\}$ is a development for a space $X$, then the obvious local bases $\{\operatorname{St}(x, G(n)): n \in N\}$ satisfy (D) because $y \in \operatorname{St}(x, G(n)) \Leftrightarrow$ $x \in \operatorname{St}(y, G(n))$ for each $x, y$ and $n$. Conversely, there exist spaces with local bases satisfying (D) which are not developable. A good example of such a space is again the "bow-tie" space of L. F. McAuley [14] (a collectionwise normal semimetric space).

This relationship between condition (D) and developability prompts a comparison of Theorem 2 with Theorem 9 (Arhangel'skii-Stone), the conditions of which may be rewritten

there exists some development $\{G(n): n \in N\}$ for $X$ such that the local bases $\{\operatorname{St}(x, G(n)): n \in N\}$ satisfy (E).

Thus, one may consider Theorem 2 to be, in some sense, strictly stronger than Theorem 9.

Recall that a space $X$ is said to be semimetric if there is some symmetric function $d$ from $X \times X$ to the nonnegative real numbers, zero on the diagonal, satisfying

$$
\inf \{d(x, y): y \in E\}=0 \text { if and only if } x \in \bar{E}
$$

for all $E \subseteq X$ and $x \in X$.

THEOREM 11. The following three conditions are equivalent for a space $X$ :

(i) $X$ is semimetric,

(ii) $X$ possesses countable local neighbourhood bases satisfying (D),

(iii) $X$ possesses countable local bases of open sets satisfying (D).

Proof. It is clearly sufficient to prove that (i) implies (iii), and that (ii) implies (i). Suppose, therefore, that $X$ possesses a semimetric $d$. For each $x \in X$ and $n \in N$ define $S(n, x)=\{y \in X: d(x, y)<1 / n\}$. It is easy to show that

(a) if $x \in U$ and $U$ is open, then there is some $n \in N$ such that $S(n, x) \subseteq U$,

(b) if $x \in X$ and $n \in N$, then $U(n, x)=S(n, x)^{\circ}$ contains. $x$. Thus, if we define

$$
R(n, x)=X \text { if } S(n, x) \nsubseteq U(m, x) \text { for each } m \in M,
$$

and otherwise

$$
\begin{gathered}
R(n, x)=U(m, x), \quad \text { where } m \text { is the largest integer less than or equal } \\
\text { to } n \text { such that } S(n, x) \subseteq U(m, x),
\end{gathered}
$$

the sets $\{R(n, x): n \in N\}$ are local bases of open sets for $X$ which satisfy (D).

Secondly, suppose that the local neighbourhood bases $\{R(n, x): n \in N\}$ satisfy (D). As in the proof of Theorem 2 we may suppose without loss of generality that 
the $\{R(n, x): n \in N\}$ are decreasing. Define a function $d: X \times X \rightarrow R$ as follows:

$$
\begin{array}{ll}
d(x, y)=0 & \text { if } x=y, \text { and otherwise, } \\
d(x, y)=1 / n, & \text { where } n \text { is the least integer such that } \\
& \text { either } x \notin R(n, y) \text { or } y \notin R(n, x) .
\end{array}
$$

It is easy to verify that $d$ is a semimetric for the space $X$.

Using this theorem, one can see, for example, from results of G. D. Creede [6, Corollary 1.4 and Theorem 2.8] that if $X$ is a space with (possibly different) sets of countable local bases of open sets satisfying (F) and (D), then the four conditions Lindelöf, $\aleph_{1}$-compact, separable, and second countable are equivalent for $X$. The category of such spaces is strictly larger than that of metrisable spaces, containing for example the space of Example 1. If the local bases satisfying (F) were chains, then metrisability would be implied by any of the four conditions above; for, $X$ would then be collectionwise normal and every second countable $T_{3}$-space is metrisable.

6. Appendix: neighbourhood axioms for metric spaces. Noting the neighbourhood characterisation of topological spaces, Theorem 1 may be rewritten as follows.

THEOREM 12. Suppose that for each $x$ in a set $X$ there is a decreasing sequence $W(x)=\{W(n, x): n=1,2, \ldots\}$ of subsets of $X$, each containing $x$, such that

(1) given $x$ and $y$ in $X, x \neq y$, there exists a positive integer $m$ with $y \notin W(m, x)$,

(2) given $x$ in $X$ and a positive integer $n$, there exist positive integers $r=r(n, x)$ and $s=s(n, x)$ such that $y \in W(r, x)$ implies that $x \in W(s, y) \subseteq W(n, x)$.

Then there is a metric for $X$ such that, for each $x$ in $X, W(x)$ is a basis for the neighbourhood system of $x$ in the metric topology.

Theorem 2 may be rewritten in a similar manner making its essential equivalence with Theorem 5 completely clear.

\section{REFERENCES}

1. P. S. Alexandroff and P. Urysohn, Une condition nécessaire et suffisante pour qu' une classe $(L)$ soit une classe (B), C. R. Acad. Sci. Paris 177 (1923), 1274-1276.

2. A. Arhangel'skii, New criteria for the paracompactness and metrizability of an arbitrary $T_{1}$-space, Soviet Math. Dokl. 2(1961), 1367-1369. (Russian)

3. R. H. Bing, Metrization of topological spaces, Canad. J. Math. 3 (1951), 175-186.

4. N. Bourbaki, General topology, Part 2, Addison-Wesley, Reading, Mass. and Hermann, Paris, 1966.

5. P. J. Collins and A. W. Roscoe, A note on metrisation (to appear).

6. G. D. Creede, Concerning semi-stratifiable spaces, Pacific J. Math. 32 (1970), 47-54.

7. R. Fox, Solution of the $\gamma$-space problem, Proc. Amer. Math. Soc. 85 (1982), 606-608.

8. A. H. Frink, Distance functions and the metrization problem, Bull. Amer. Math. Soc. 43 (1937), $133-142$.

9. S. Hanai, On closed mappings, Proc. Japan Acad. 30 (1954), 285-288.

10. R. W. Heath, D. J. Lutzer and P. L. Zenor, Monotonically normal spaces, Trans. Amer. Math. Soc. 178 (1973), 481-493.

11. R. E. Hodel, Spaces defined by sequences of open covers which guarantee that certain sequences have cluster points, Duke Math. J. 39 (1972), 253-263.

12. W. F. Lindgren and P. Fletcher, Locally quasi-uniform spaces with countable bases, Duke Math. J. 41 (1974), 231-240. 
13. W. F. Lindgren and P. J. Nyikos, Spaces with bases satisfying certain order and intersection properties, Pacific J. Math. 66 (1976), 455-476.

14. L. F. McAuley, A relation between perfect separability, completeness, and normality in semimetric spaces, Pacific J. Math. 6 (1956), 315-326.

15. E. Michael, Yet another note on paracompact spaces, Proc. Amer. Math. Soc. 10 (1959), 309-314.

16. The product of a normal space and a metric space need not be normal, Bull. Amer. Math. Soc. 69 (1963), 375-376.

17. R. L. Moore, $A$ set of axioms for plane analysis situs, Fund. Math. 25 (1935), 13-28.

18. K. Morita, On the simple extension of a space with respect to a uniformity. IV, Proc. Japan Acad. 27 (1951), 632-636.

19. J. Nagata, On a necessary and sufficient condition for metrizability, J. Inst. Polytech. Osaka City Univ. Ser. A Math. 1 (1950), 93-100.

20. 8 (1957), 185-192.

21. Modern general topology, Wolters-Noordhoff, Groningen, 1968.

22. V. W. Niemytzki, On the 'third axiom of metric space', Trans. Amer. Math. Soc. 29 (1927), 507-513.

23. P. Roy, Failure of equivalence of dimension concepts for metric spaces, Bull. Amer. Math. Soc. 68 (1962), 609-613.

24. M. E. Rudin, A new proof that metric spaces are paracompact, Proc. Amer. Math. Soc. 20 (1969), 603.

25. Yu. M. Smirnov, A necessary and sufficient condition for metrizability of topological spaces, Dokl. Akad. Nauk SSSR 77 (1951), 197-200. (Russian)

26. A. H. Stone, Paracompactness and product spaces, Bull. Amer. Math. Soc. 54 (1948), 977-982.

27. Sequences of coverings, Pacific J. Math. 10 (1960), 689-691.

28. J. Suzuki, On the metrization and completion of a space with respect to a uniformity, Proc. Japan Acad. 27 (1951), 219-223.

29. J. Williams, Locally uniform spaces, Trans. Amer. Math. Soc. 168 (1972), 435-469.

St. Edmund Hall, OXford, OXI 4AR, U. K.

UNIVERSITY COLLEGE, OXFORD, OXI 4BH, U. K. 